\section{The hormone trigger in PTSD}

\section{By Lev Osherovich, Senior Writer}

Post-traumatic stress disorder is thought to involve changes in the circuitry of brain regions related to fear, although the specific molecular players have been elusive. Now, U.S. researchers have evidence that dysregulation of a brain hormone called PACAP and its receptor, PAC1, contributes to the disorder in women. ${ }^{1}$

Post-traumatic stress disorder (PTSD) is a syndrome of anxiety and emotional disturbance following traumatic experiences such as combat and is often confused with depression. PTSD typically is treated with talk therapy and anxiolytics.

Adenylate cyclase activating polypeptide pituitary (ADCYAP1; PACAP) and its receptor, adenylate cyclase activating polypeptide 1 pituitary receptor type I (ADCYAP1R1; PAC1), relay signals in the hypothalamus and the amygdala, the brain region in which emotions originate.

The new findings, from a team led by researchers at Emory University and The University of Vermont, draw on mouse studies and human genetic and biochemical data to suggest that excessive PACAP-PAC1 activity leads to PTSD. The results open the door to identifying biomarkers and therapeutics to selectively diagnose and treat PTSD.

"From the standpoint of understanding the molecular mechanism of PTSD, this work is revolutionary," said Anatoly Kreinin, director of the psychiatric department at Tirat Carmel Mental Health Center. "This is one of those rare psychiatric disorders where we now know the trigger."

\section{Blue gene}

The group's biochemical and genetic studies of a cohort of highly traumatized women converged on the PACAP-PAC1 pathway.

"We were looking for genes associated with PTSD in a small genomewide association study," said team leader Kerry Ressler, associate professor of psychiatry and behavioral sciences at Emory. "At the same time, we were looking at gene expression in the amygdala. Our hypothesis was that there would be different pathways expressed in fear learning in the amygdalas" of PTSD patients compared with in those of healthy controls.

In the genomewide association study and a larger follow-up study, a polymorphism near the PAC1 gene correlated with PTSD in women but not men. In a retrospective analysis, homozygous female carriers of the risk allele had greater arousal and startle responses than heterozygotes or noncarriers.

At the same time, the team looked at differences in PACAP levels in the serum of traumatized women and men deemed to be at risk for PTSD. PACAP levels were higher in women who went on to develop PTSD than in those who did not, suggesting that increased PACAP is a biomarker for the disorder. The team found no correlation between PACAP levels and PTSD symptoms in men.

The reason for the female-specific PACAP-PAC1 dysregulation remains a mystery. A potential explanation is that expression of the receptor appears to be regulated by the female sex hormone estrogen. Indeed, the team found that rats treated with estrogen had higher levels of Pacl mRNA than vehicle-treated controls.

Collectively, the findings suggested that either an excess of PACAP or a polymorphism in the gene for PAC1 could lead to PTSD.

To test these hypotheses, the team turned to a mouse model of the disorder. In a classical aversive conditioning experiment in which mice were taught to fear a sound that accompanied an electric shock, conditioned mice indeed had higher levels of Pac1 mRNA in the amygdala than unconditioned controls.

Results were reported in Nature. The team also included researchers from the Yerkes National Primate Research Center at Emory University and the University of Miami.

Guy Griebel, senior scientist in the Exploratory Unit of sanofi-aventis Group, said it is unclear whether the team's rodent models of anxiety accurately reflect the deep-rooted, long-term fear that characterizes PTSD.

"PTSD is a disease that develops over time, where you have a traumatic event and several weeks later you have the disorder," he said. "Most of the models in this study used acute stress. They should check behavior two or four weeks afterward" to confirm that PACAP activity leads to long-term anxiety.

sanofi-aventis' SAR125543, an antagonist of corticotropin-releasing factor 1 (CRF1), is in Phase II development for major depressive disorder and PTSD.

\section{Fear factor}

Victor May, professor of anatomy and neurobiology at the University of Vermont and a coauthor of the study, said the findings were in line with previous rodent work from his lab and others that suggested the PACAP-PAC1 pathway is a critical mediator of fear.

"We found that when you make rats extremely anxious, you get a 10 -fold increase in PACAP in the bed nucleus of the stria terminalis, which is the nexus of all of the anxiety pathways before they enter the hypothalamus," he said. "If you knock out PACAP or PAC1 in mice, those mice are less anxious" than wild-type controls.

Thus, concluded May, "a PAC1 antagonist could be a potential way to treat or possibly prevent PTSD." 
Proving that the PACAP-PAC1 pathway is druggable will require further genetic and pharmacological work in rodents.

Albert Pinhasov, chairman of the Department of Molecular Biology at Ariel University Center of Samaria, said PACAP is one of many neuropeptide signaling systems that form a complex network responsible for fine-tuning emotional behavior. These other signaling systems include corticotropin-releasing factor (CRF), neuropeptide Y (NPY), calcitonin and neurokinin 1 Substance P (TAC1; NK2).

"In many different regions, these neuropeptide systems act as modulators of neurons that affect the synthesis of other neuropeptides," he said. "They probably all work together," the PACAP system included.

Because the effect of inhibiting PACAP signaling outright may affect many brain pathways, Pinhasov and Kreinin think it would be better to screen for compounds that modulate or partially antagonize PAC1.

The two researchers said they know of undisclosed pharmaceutical companies working on partial antagonists but said the screens have not yielded suitable compounds.

For companies that opt to try blocking PAC1, Ressler said it will be important to characterize how the receptor is regulated.

"While there's probably an increase in PACAP hormone levels in some PTSD patients, it gets more complicated with the receptor," said Ressler. "The PTSD-linked polymorphism is in the regulatory region of the receptor gene, but we don't really know if it's increasing or decreasing" PACAP-PAC1 signaling in carriers of the risk allele.

\section{Climate of fear}

Other targeted approaches to PTSD in clinical testing include GSK561679, a corticotropin-releasing factor receptor 1 (CRHR1; CRFR1) antagonist from GlaxoSmithKline plc and Neurocrine Biosciences Inc. It is in Phase II testing.

GSK also is developing orvepitant (GW823296), a neurokinin 1 Substance P receptor (TACR1) antagonist that is in Phase I trials.

According to GSK spokesperson Melinda Stubbee, the future of both compounds is under review as part of GSK's 2010 announcement that it is exiting several areas of neuroscience including pain, anxiety and depression.
Therapeutic applications aside, Ressler and May noted that monitoring PACAP levels in blood could help in the differential diagnosis of PTSD from other anxiety disorders. They noted that patients with general depression or bipolar disorder did not have high PACAP, suggesting that the hormone could be a biomarker specific to PTSD.

Griebel thinks the PAC1 polymorphism also could be useful in the design of clinical trials.

He said screening trial enrollees for the PTSD-linked polymorphism before PTSD symptoms occur could enable a prevention trial, something that hasn't yet been attempted. Alternatively, "it would be interesting to see if PACAP elevation could serve as a diagnostic or biomarker for successful treatment."

Griebel said that developing a robust procedure for measuring blood PACAP would let researchers validate the correlation between PACAP and PTSD in a broader population of patients including combat veterans.

For now, the hormone appears to be a good biomarker for PTSD in traumatized women, "but perhaps in a different population this may not be such a useful marker," he noted.

Ressler and May are trying to replicate their findings in other patient cohorts and are refining their diagnostic procedures. They have filed patents on diagnostic and therapeutic applications of their discoveries. These patents are available for licensing from the University of Vermont.

Osherovich, L. SciBX 4(11); doi:10.1038/scibx.2011.301

Published online March 17, 2011

\section{REFERENCES}

1. Ressler, K.J. et al. Nature; published online Feb. 24, 2011; doi:10.1038/nature09856

Contact: Kerry J. Ressler, Emory University, Atlanta, Ga. e-mail: kressle@emory.edu

\section{COMPANIES AND INSTITUTIONS MENTIONED}

Ariel University Center of Samaria, Ariel, Israel

Emory University, Atlanta, Ga.

GlaxoSmithKline plc (LSE:GSK; NYSE:GSK), London, U.K. Neurocrine Biosciences Inc. (NASDAQ:NBIX), San Diego, Calif. sanofi-aventis Group (Euronext:SAN; NYSE:SNY), Paris, France Tirat Carmel Mental Health Center, Tirat Carmel, Israel University of Miami, Miami, Fla.

The University of Vermont, Burlington, Vt. Yerkes National Primate Research Center at Emory University, Atlanta, Ga. 